Supporting Information for

\title{
Covalently bonded sulfur anchored with thiol- modified carbon nanotube as a cathode material for lithium-sulfur batteries
}

Ya-Wen Xu, Bo-Hai Zhang, Guo-Ran Li, Sheng Liu* and Xue-Ping Gao

Institute of New Energy Material Chemistry, School of Materials Science and

Engineering, Nankai University, Tianjin 300350, China.

E-mail: shengliu@nankai.edu.cn. 

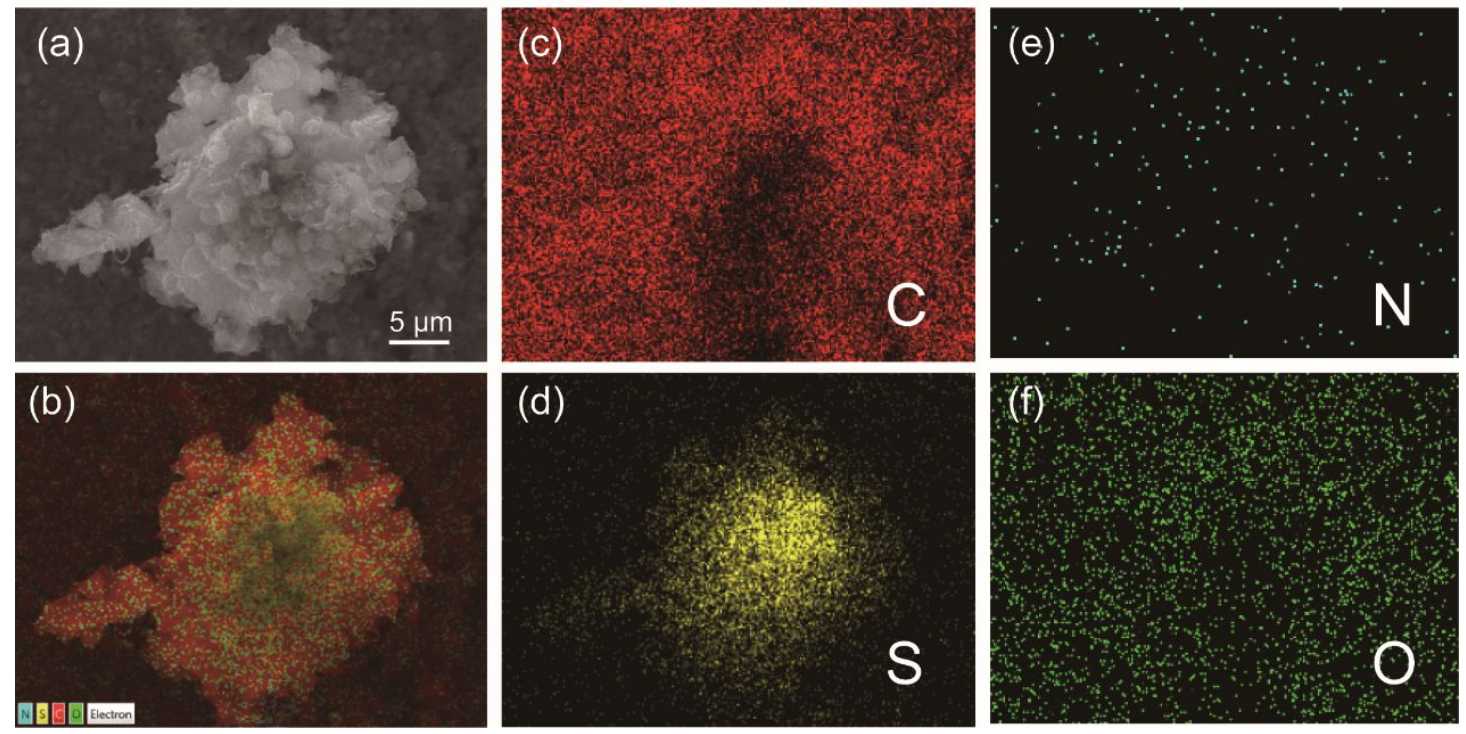

Figure S1 Characterization of the as-prepared CNTSH/S. (a) SEM images of CNTSH/S. Elemental mappings of (b) composite element, (c) C, (d) S, (e) N and (f) $\mathrm{O}$.

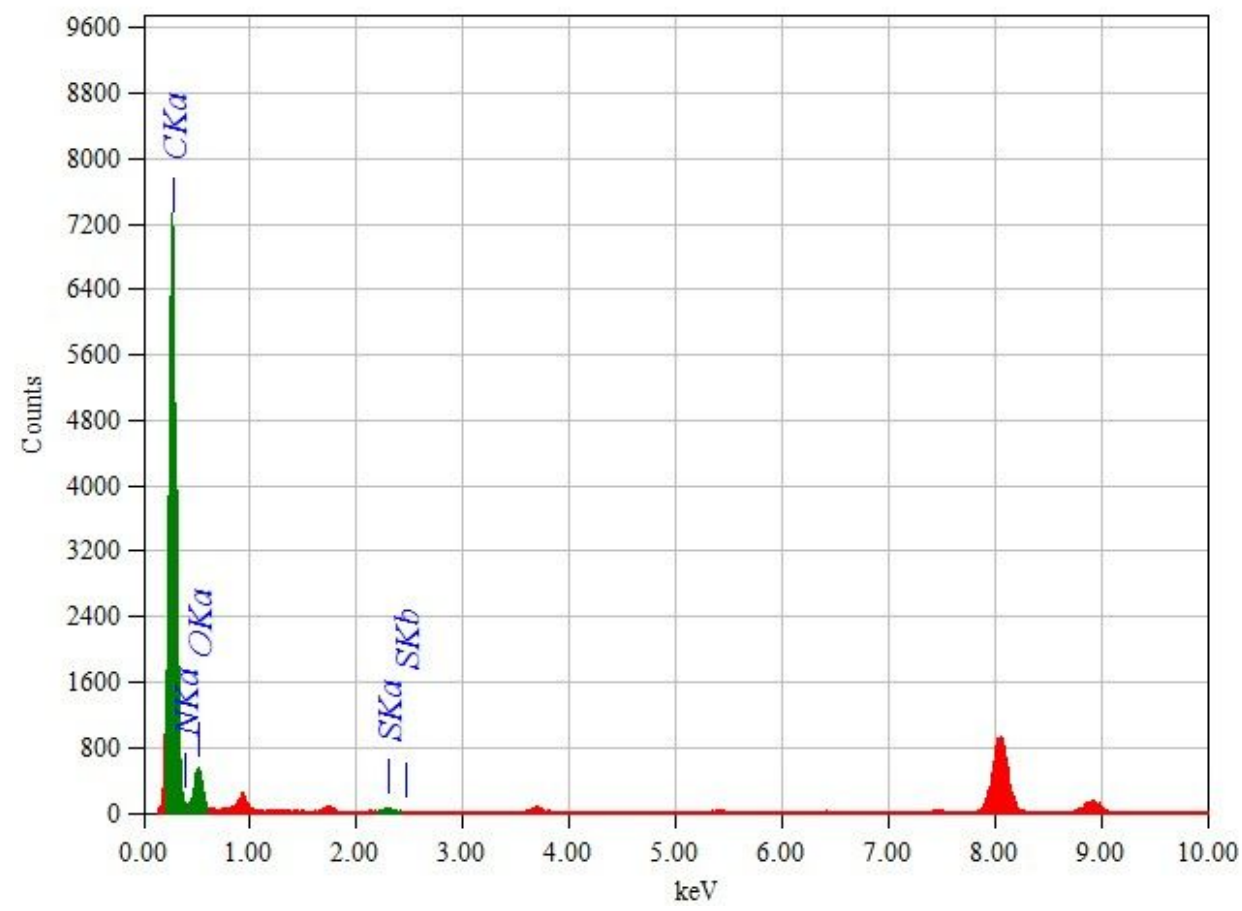

Figure S2 Energy dispersive spectroscopy result of the CNTSH 


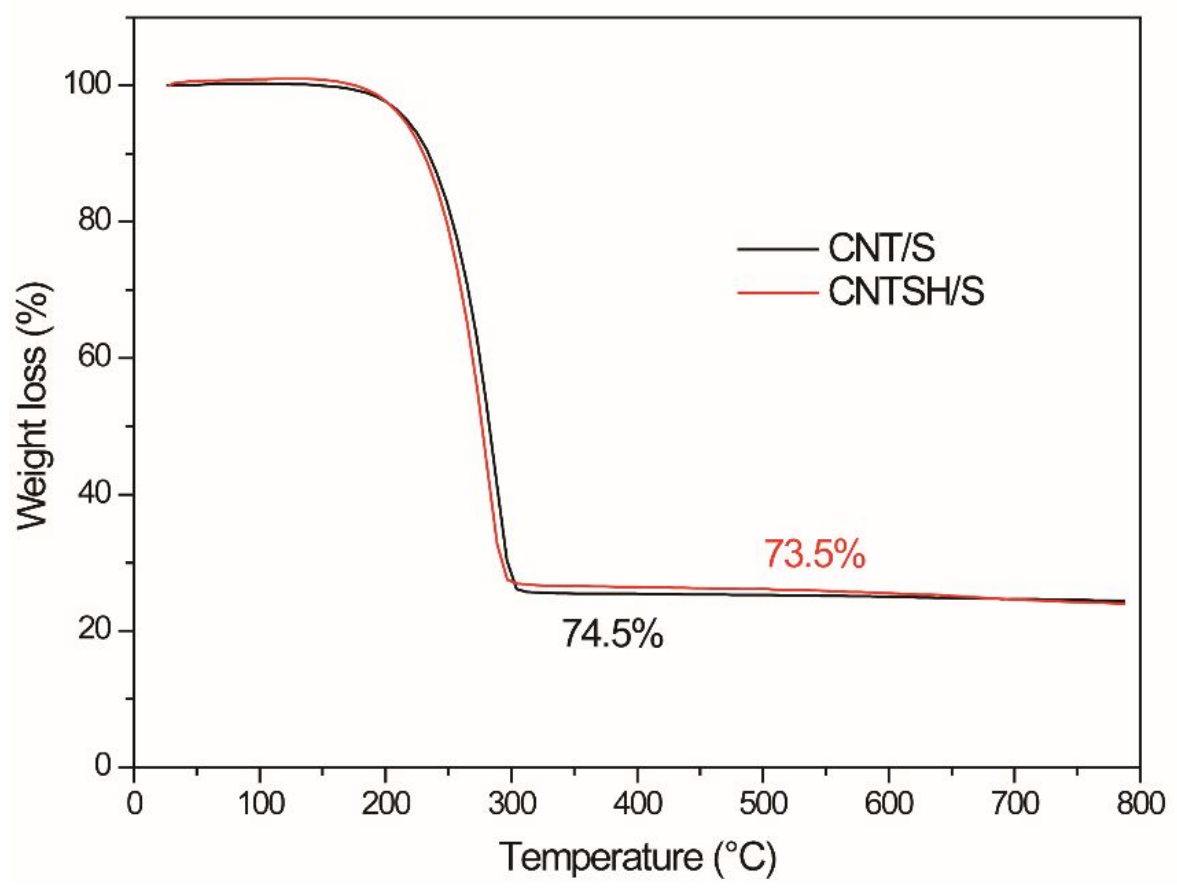

Figure S3 TG profiles of the as-prepared CNTSH/S and CNT/S composite. 


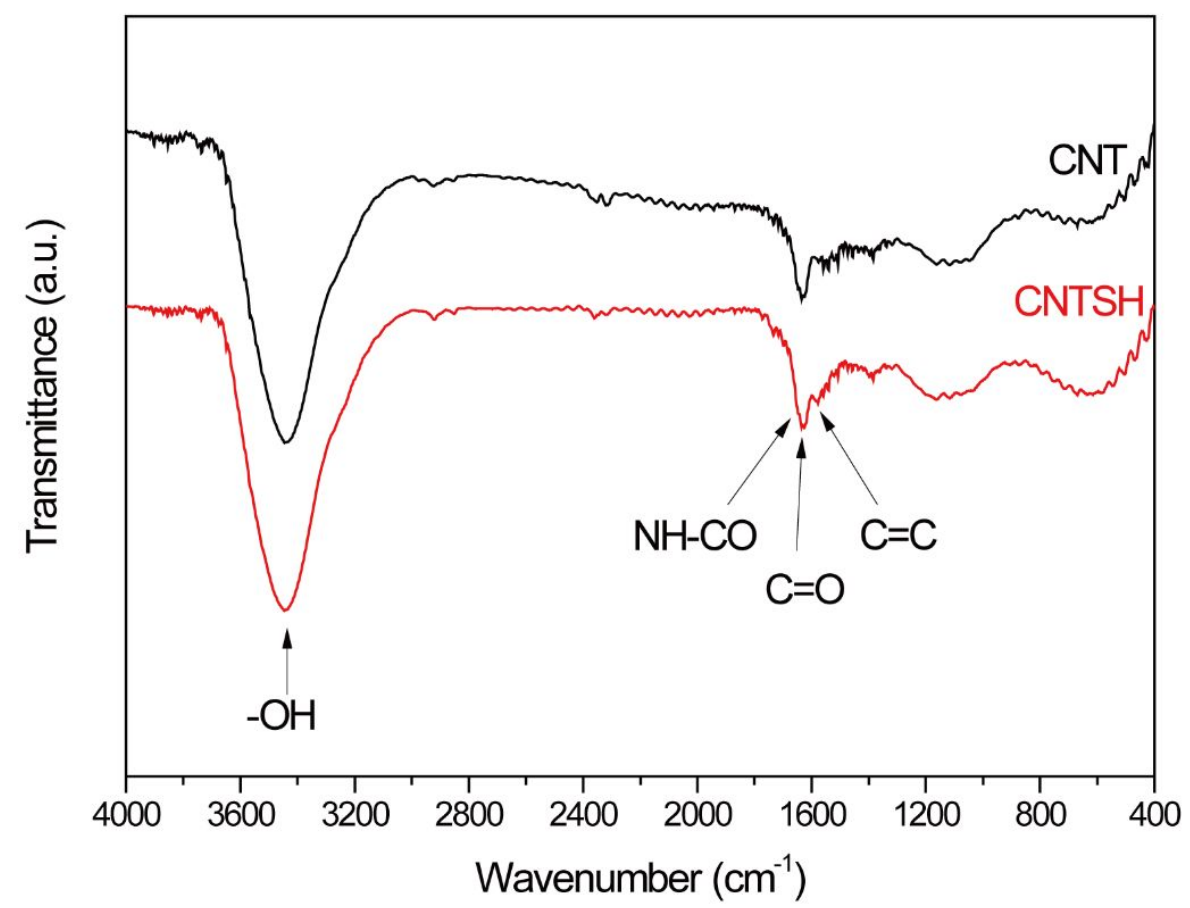

Figure S4 FTIR spectra for the CNTSH and CNT

(a)

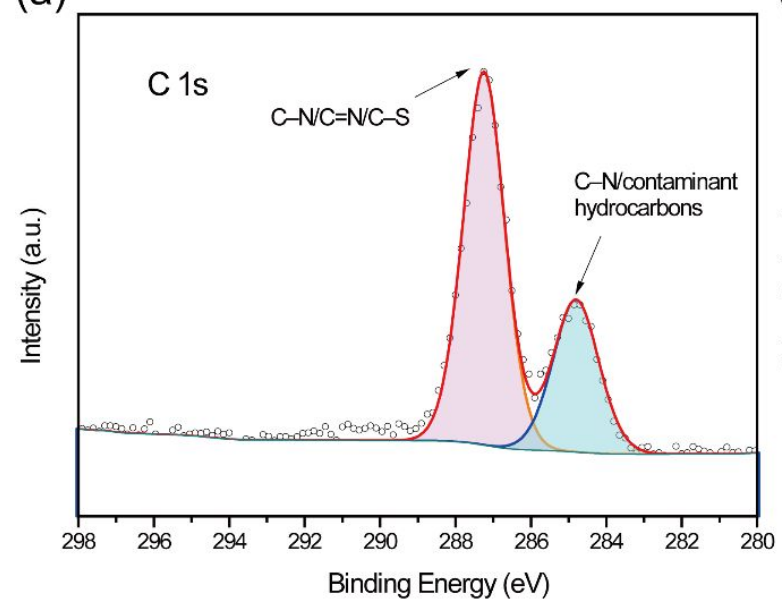

(b)

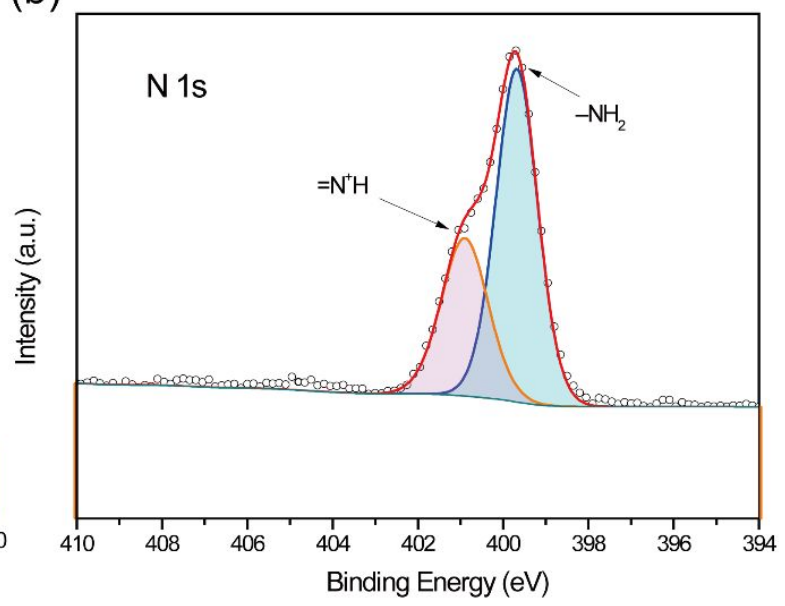

Figure S5 XPS spectra of 5-amino-1,3,4-thiadiazole-2-thiol: (a) C 1s region, (b) N 1s region 


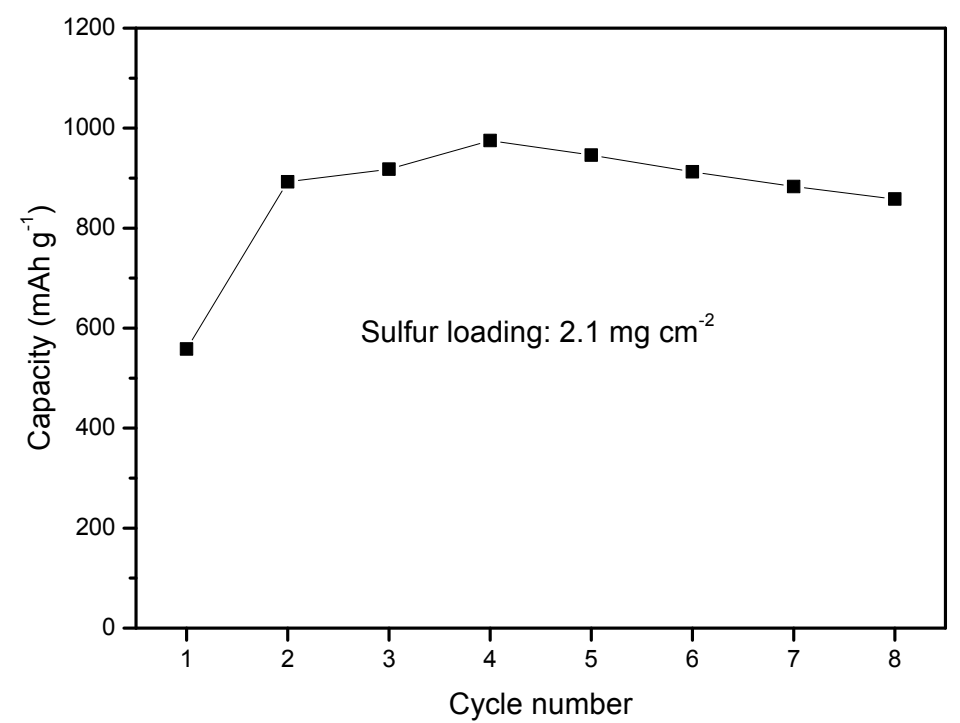

Figure S6 Electrochemical performance of the $\mathrm{CNTSH} / \mathrm{S}$ cathode with sulfur loading of $2.1 \mathrm{mg} \mathrm{cm}^{-2}$ at $0.1 \mathrm{C}$ rate.

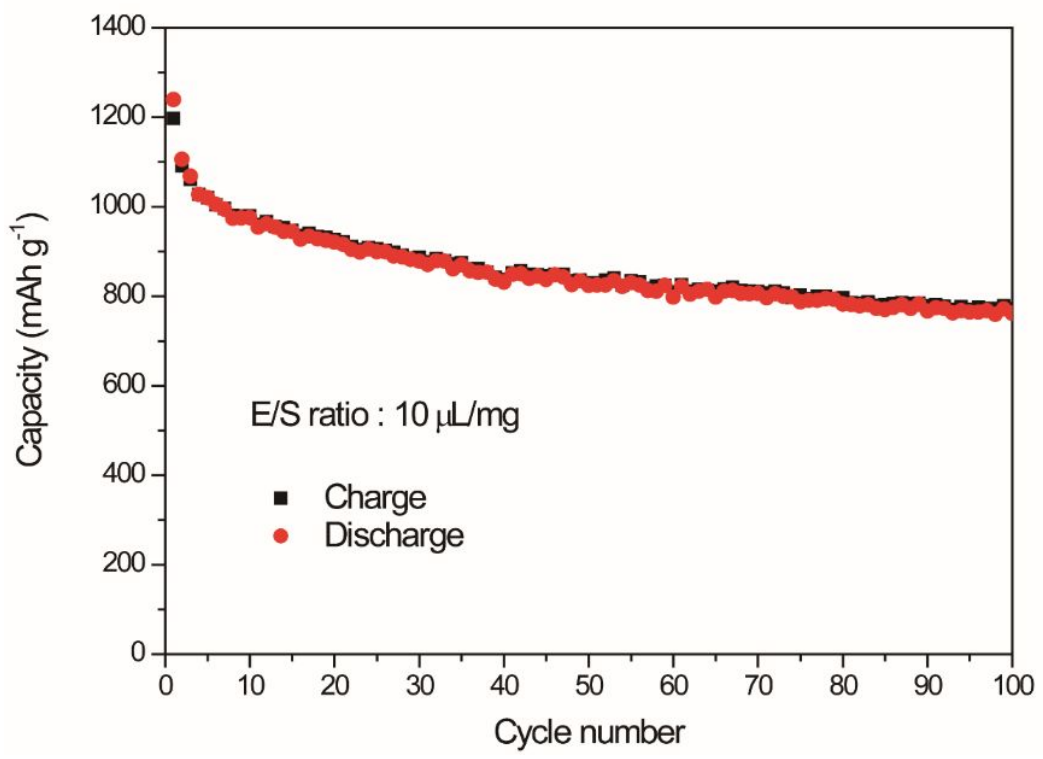


Figure S7 The cycling performance of the CNTSH/S electrode with low electrolyte/sulfur ratios $(\mathrm{E} / \mathrm{S})$ at $0.1 \mathrm{C}$ rate

Table S1 Energy dispersive spectroscopy result of the CNTSH

\begin{tabular}{lccccc}
\hline Element & $(\mathrm{keV})$ & Mass\% & Counts & Sigma & Atom\% \\
\hline C K (Ref.) & 0.277 & 94.28 & 66411.39 & 0.35 & 95.55 \\
N K & 0.392 & 2.20 & 2291.62 & 0.07 & 1.91 \\
O K & 0.525 & 3.16 & 4356.28 & 0.08 & 2.40 \\
S K & 2.307 & 0.36 & 444.02 & 0.04 & 0.14 \\
Total & & 100.00 & & & 100.00 \\
\hline
\end{tabular}

Table S2 The comparison of electrochemical performance between CNTSH/S cathode and related reported works.

\begin{tabular}{cccccc}
\hline Sample & $\begin{array}{c}\text { Current } \\
\text { density }\end{array}$ & Initial capacity & $\begin{array}{c}\text { Cycle } \\
\text { number }\end{array}$ & $\begin{array}{c}\text { Capacity (final } \\
\text { cycle) }\end{array}$ & Ref. \\
\hline CNTSH/S & $0.1 \mathrm{C}$ & $1337.6 \mathrm{~mA} \mathrm{~h} \mathrm{~g}^{-1}$ & 300 & $669.2 \mathrm{~mA} \mathrm{~h} \mathrm{~g}^{-1}$ & This work \\
S/MWNT-OH25 & - & $1274 \mathrm{~mA} \mathrm{~h} \mathrm{~g}^{-1}$ & 100 & $721.0 \mathrm{~mA} \mathrm{~h} \mathrm{~g}^{-1}$ & {$[1]$} \\
Sulfur electrode with MWNT & - & $485 \mathrm{~mA} \mathrm{~h} \mathrm{~g}^{-1}$ & 50 & $300 \mathrm{~mA} \mathrm{~h} \mathrm{~g}^{-1}$ & {$[2]$} \\
S@CNT-g-Gr & $0.2 \mathrm{C}$ & $1200 \mathrm{~mA} \mathrm{~h} \mathrm{~g}^{-1}$ & 350 & $800 \mathrm{~mA} \mathrm{~h} \mathrm{~g}^{-1}$ & {$[3]$} \\
S/CPS-70 & $0.5 \mathrm{C}$ & $950 \mathrm{~mA} \mathrm{~h} \mathrm{~g}^{-1}$ & 300 & $750 \mathrm{~mA} \mathrm{~h} \mathrm{~g}^{-1}$ & {$[4]$}
\end{tabular}




\section{Supplementary References}

[1] Kim, J. H.; Fu, K.; Choi, J.; Sun, S.; Kim, J.; Hu, L.; Paik, U. Hydroxylated Carbon Nanotube Enhanced Sulfur Cathodes for Improved Electrochemical Performance of Lithium-Sulfur Batteries. Chem. Commun. 2015, 51, 1368213685.

[2] Han, S. C.; Song, M. S.; Lee, H.; Kim, H. S.; Ahn, H. J.; Lee, J. Y. Effect of Multiwalled Carbon Nanotubes on Electrochemical Properties of Lithium/Sulfur Rechargeable Batteries. J. Electrochem. Soc. 2003, 150, A889-A893.

[3] Wang, Q. Q.; Huang, J. B.; Li, G. R.; Lin, Z.; Liu, B. H.; Li, Z. P. A Facile and Scalable Method to Prepare Carbon Nanotube-Grafted-Graphene for High Performance Li-S Battery. J. Power Sources 2017, 339, 20-26.

[4] Ma, L.; Zhuang, H. L.; Wei, S.; Hendrickson, K. E.; Kim, M. S.; Cohn, G.; Hennig, R. G.; Archer, L. A. Enhanced Li-S Batteries Using AmineFunctionalized Carbon Nanotubes in the Cathode. ACS Nano 2016, 10, 10501059. 
[5] Ponraj, R.; Kannan, A. G.; Ahn, J. H.; Lee, J. H.; Kang, J.; Han, B.; Kim, D. W. Effective Trapping of Lithium Polysulfides Using a Functionalized Carbon Nanotube-Coated Separator for Lithium-Sulfur Cells with Enhanced Cycling Stability. ACS Appl. Mater. Interfaces 2017, 9, 38445-38454.

[6] Wang, Z.; Dong, Y.; Li, H.; Zhao, Z.; Wu, H. B.; Hao, C.; Liu, S.; Quu, J.; Lou, X. W. Enhancing Lithium-Sulphur Battery Performance by Strongly Binding the Discharge Products on Amino-Functionalized Reduced Graphene Oxide. Nat. Commun. 2014, 5, 5002. 